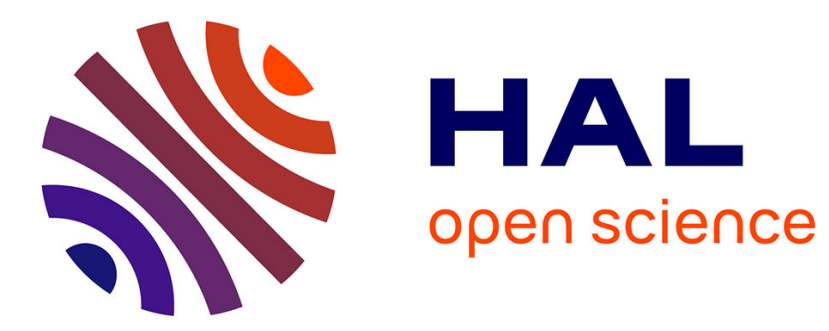

\title{
Comportement mécanique de poutres attaquées par la corrosion
}

\author{
Ph Turcry, Stéphanie Bonnet, Gilles Pijaudier-Cabot
}

\section{To cite this version:}

Ph Turcry, Stéphanie Bonnet, Gilles Pijaudier-Cabot. Comportement mécanique de poutres attaquées par la corrosion: Utilisation du code de calcul Eficos. Revue Européenne de Génie Civil, 2007, 11 (1-2), pp.195-211. 10.1080/17747120.2007.9692928 . hal-01006967

\section{HAL Id: hal-01006967 https://hal.science/hal-01006967}

Submitted on 26 Mar 2017

HAL is a multi-disciplinary open access archive for the deposit and dissemination of scientific research documents, whether they are published or not. The documents may come from teaching and research institutions in France or abroad, or from public or private research centers.
L'archive ouverte pluridisciplinaire HAL, est destinée au dépôt et à la diffusion de documents scientifiques de niveau recherche, publiés ou non, émanant des établissements d'enseignement et de recherche français ou étrangers, des laboratoires publics ou privés. 


\section{Comportement mécanique de poutres attaquées par la corrosion}

\section{Utilisation du code de calcul Eficos}

\section{Philippe Turcry — Stéphanie Bonnet — Gilles Pijaudier-Cabot}

Institut de recherche en génie civil et mécanique (GeM)

Ecole Centrale de Nantes

1 rue de la Nö̈, F-44321 Nantes cedex 3

stephanie.bonnet@univ-nantes.fr

RÉSUMÉ. Cette étude a pour but de simuler le comportement mécanique des poutres de la Rance qui ont été immergées pendant 40 ans en eau de mer. Ces poutres après auscultation ont été testées mécaniquement en flexion 4-points et en traction directe. Notre travail porte sur la modélisation du comportement mécanique des poutres avant et après corrosion à l'aide du code de calcul Eficos. La prise en compte de la corrosion est limitée aux pertes de sections des aciers qui sont des ronds lisses. On obtient une bonne adéquation entre simulations et résultats expérimentaux, en particulier dans le cas de la flexion en configuration béton armé.

ABSTRACT. The aim of this study is to predict the mechanical behaviour of beams which were in sea water for 40 years. Mechanical experimentations were carried out after inspection of beams: loading in four-point bending and loading in direct tension. The mechanical behaviour of the beams is modelled before and after being corroded, with the software Eficos. The steel corrosion is taken into account only by a reduction in steel cross section because steels have no anchorage. A good correlation is obtained between simulations and experimental results for bending in the case of reinforced concrete.

MOTS-CLÉS : poutre béton armé, précontrainte, corrosion, flexion, traction. KEYWORDS: reinforced concrete beams, prestressing, corrosion, bending, tension. 


\section{Introduction}

Une des préoccupations actuelles des maîtres d'ouvrage de structures en environnement marin est de connaître l'influence de la corrosion des armatures sur la résistance mécanique de l'ouvrage. Ces informations sont importantes pour le choix d'une stratégie de maintenance de ces ouvrages. Un besoin croissant de modèle de vieillissement avec prise en compte de la corrosion est donc identifié. Le benchmark des poutres de la Rance donnera des éléments d'évaluation de certains modèles existants.

Notre contribution à ce benchmark porte donc sur la modélisation mécanique du comportement des poutres après 40 ans d'immersion en eau de mer. Notre objectif est d'estimer dans le sens de la sécurité le comportement mécanique des poutres en flexion et traction en prenant en compte l'effet de la corrosion des aciers. Les comportements mécaniques ainsi modélisés seront comparés aux courbes obtenues expérimentalement au CEBTP et également aux autres modélisations.

Après une présentation du code de calcul utilisé (Eficos) et des hypothèses, nous aborderons l'influence du maillage utilisé et des conditions aux limites. Enfin, les résultats obtenus seront présentés dans une dernière partie.

\section{Présentation du modèle Eficos}

\subsection{Principe de la modélisation}

Formulé dans le cadre de la mécanique des milieux continus, Eficos est un code aux éléments finis, développé pour prédire le comportement de poutres en béton armé ou non en flexion ou traction (Bazant et al., 1987 ; Laborderie, 1991 ; Daniel, 2001).

Eficos est basé sur la théorie des poutres de Bernoulli. Ses éléments, en 1 dimension avec 3 degrés de liberté, sont discrétisés en couches, qui permettent de prendre en compte par homogénéisation la présence des armatures (figure 1). L'homogénéisation s'effectue sur le module sécant de la couche, calculé comme la somme des modules sécants de l'acier et du béton pondérés par leur surface respective (équation [1]) :

$$
\mathrm{S}_{\mathrm{t}} * \mathrm{E}=\mathrm{S}_{\mathrm{b}} * \mathrm{E}_{\mathrm{b}}+\mathrm{S}_{\mathrm{a}} * \mathrm{E}_{\mathrm{a}}
$$

Dans le cadre du BMC Rance, un modèle de précontrainte a été ajouté au code. La précontrainte des poutres de la Rance est obtenue par post-tension de câbles placés dans des gaines traversant la poutre. C'est pourquoi nous avons choisi de modéliser la précontrainte comme un simple effort extérieur. Cet effort est appliqué aux extrémités de la poutre avec une excentricité égale à celle des câbles (excentricité constante suivant la longueur de la poutre). Sa valeur initiale est égale 
à l'effort de précontrainte mesuré lors de l'auscultation des poutres (donnée du benchmark). La variation de l'effort de précontrainte en cours de chargement est modélisée en considérant la variation de longueur de la couche dans lequel se situe les câbles. L'effort de précontrainte évolue donc avec cette longueur, pondéré par la section des câbles et leur module élastique (on suppose que la limite élastique des câbles est suffisamment élevée pour que les câbles restent élastiques).

Le modèle de comportement du béton, détaillé ci-après, est un modèle d'endommagement non local. Les simulations nous donnent donc le comportement mécanique global des poutres, mais non une cartographie de la fissuration. Les simulations sont par ailleurs effectuées uniquement pour un chargement monotone.
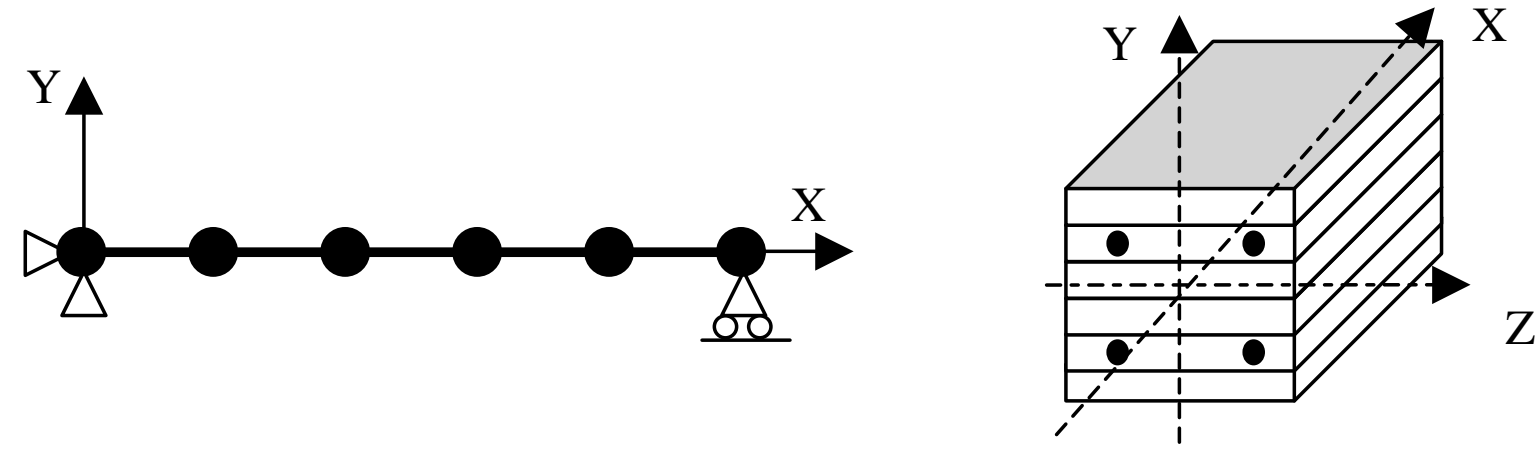

Figure 1. Discrétisation de la poutre en éléments et des éléments en couches

\subsection{Comportement du béton}

Le modèle utilisé est le modèle d'endommagement isotrope non local développé par Pijaudier-Cabot et Mazars (Pijaudier-Cabot et al., 2001). L'effet mécanique de la micro-fissuration due au chargement extérieur est décrit par une variable scalaire $d$. Cette dernière varie entre 0 pour le matériau sain et 1 pour la rupture.

$$
\sigma_{\mathrm{ij}}=(1-\mathrm{d}) \Lambda_{\mathrm{ijkl}} \varepsilon_{\mathrm{kl}}
$$

où $\sigma_{i j}$ et $\varepsilon_{i j}$ sont respectivement les composantes du tenseur des contraintes et du tenseur des déformations. $\Lambda_{i j k l}$ sont les coefficients d'élasticité du matériau non endommagé. Pour décrire la variation de l'endommagement on définit tout d'abord la déformation équivalente $\tilde{\varepsilon}$ :

$$
\tilde{\varepsilon}=\sqrt{\sum_{i=1}^{3}\left(\left\langle\varepsilon_{i}\right\rangle_{+}\right)^{2}}
$$

$\left\langle\varepsilon_{i}\right\rangle_{+}=0$ si $\varepsilon_{i}<0$ et $\left\langle\varepsilon_{i}\right\rangle_{+}=\varepsilon_{i}$ si $\varepsilon_{i} \geq 0 ; \quad \varepsilon_{i}$ représente les déformations principales. Dans l'approche non locale, la variable contrôlant l'endommagement est la moyenne de cette déformation équivalente : 


$$
\bar{\varepsilon}(\mathrm{x})=\frac{1}{\mathrm{~V}_{\mathrm{r}}(\mathrm{x})} \int_{\Omega} \psi(\mathrm{x}-\mathrm{s}) \widetilde{\varepsilon}(\mathrm{s}) \mathrm{ds} \text { avec } \mathrm{V}_{\mathrm{r}}(\mathrm{x})=\int_{\Omega} \psi(\mathrm{x}-\mathrm{s}) \mathrm{ds}
$$

où $\Omega$ est le volume de la structure, $V_{r}(x)$ est le volume représentatif au point $x$, et $\psi(x-s)$ est la fonction de pondération non locale :

$$
\psi(\mathrm{x}-\mathrm{s})=\exp \left(-\frac{4\|\mathrm{x}-\mathrm{s}\|^{2}}{1_{c}^{2}}\right)
$$

$l_{c}$ est la longueur interne du matériau. Ce paramètre central dans la modélisation du comportement mécanique sera discuté par la suite. La déformation moyenne équation [4] contrôle l'accroissement de l'endommagement sous les conditions suivantes :

$$
\begin{aligned}
& \mathrm{F}(\bar{\varepsilon})=\bar{\varepsilon}-\kappa \\
& \text { si } \mathrm{F}(\bar{\varepsilon})=0 \text { et } \dot{\mathrm{F}}(\bar{\varepsilon})=0 \text { alors }\left\{\begin{array} { l } 
{ \mathrm { d } = \mathrm { h } ( \kappa ) } \\
{ \kappa = \overline { \varepsilon } }
\end{array} \text { avec } \dot { \mathrm { d } } \geq 0 \text { sinon } \left\{\begin{array}{l}
\dot{\mathrm{d}}=0 \\
\dot{\kappa}=0
\end{array}\right.\right.
\end{aligned}
$$

La surface seuil est définie par $F(\bar{\varepsilon})=0$ et $\dot{d} \geq 0$. Initialement, $\kappa=\kappa_{0}$, où $\kappa_{0}$ est la déformation à laquelle s'amorce l'endommagement. Cette déformation est usuellement celle mesurée au pic en contrainte lorsque le matériau est sollicité en traction simple :

$$
\kappa_{0}=\frac{f_{t}}{E}
$$

$f_{t}$ est la contrainte maximale de traction. Dans la loi d'évolution $h(\kappa)$, l'endommagement est décomposé en deux parties, $d_{t}$ pour la traction et $d_{c}$ pour la compression :

$$
\left\{\begin{array}{l}
d_{t}=1-\frac{\kappa_{0}\left(1-A_{t}\right)}{\kappa}-\frac{A_{t}}{\exp \left(B_{t}\left(\kappa-\kappa_{0}\right)\right)} \\
d_{c}=1-\frac{\kappa_{0}\left(1-A_{c}\right)}{\kappa}-\frac{A_{c}}{\exp \left(B_{c}\left(\kappa-\kappa_{0}\right)\right)} \\
d=\alpha_{t} d_{t}+\alpha_{c} d_{c}
\end{array}\right.
$$

$A_{t}, B_{t}, A_{c}$ et $B_{c}$ sont les paramètres du modèle. Pour des bétons classiques :

$$
1 \leq A_{c} \leq 1.5, \quad 10^{3} \leq B_{c} \leq 2.10^{3}, \quad 0.7 \leq A_{t} \leq 1.2, \quad 10^{4} \leq B_{t} \leq 5.10^{4}
$$


Les coefficients $\alpha_{c}$ et $\alpha_{t}$ sont déduits des déformations principales décomposées en une déformation de traction (indice t) et de compression (indice c) :

$$
\begin{aligned}
& \varepsilon_{i}=\varepsilon_{i}^{t}+\varepsilon_{i}^{c} \\
& \operatorname{avec}\left\{\begin{array}{l}
\varepsilon_{i j}^{t}=(1-d) \Lambda_{i j k l}^{-1} \sigma_{k l}^{t} \\
\varepsilon_{i j}^{c}=(1-d) \Lambda_{i j k l}^{-1} \sigma_{k l}^{c}
\end{array}\right.
\end{aligned}
$$

et

$$
\alpha_{\mathrm{t}}=\sum_{\mathrm{i}=1}^{3}\left(\frac{\left\langle\varepsilon_{\mathrm{i}}^{\mathrm{t}}\right\rangle_{+}\left\langle\varepsilon_{\mathrm{i}}\right\rangle_{+}}{\widetilde{\varepsilon}^{2}}\right), \quad \alpha_{\mathrm{c}}=\sum_{\mathrm{i}=1}^{3}\left(\frac{\left\langle\varepsilon_{\mathrm{i}}^{\mathrm{c}}\right\rangle_{+}\left\langle\varepsilon_{\mathrm{i}}\right\rangle_{+}}{\widetilde{\varepsilon}^{2}}\right)
$$

$\sigma^{t}$ et $\sigma^{c}$ sont respectivement les parties positives et négatives du tenseur des contraintes (décomposition modale).

Cette loi de comportement est employée dans sa version uniaxiale. En traction, la déformation équivalente est égale à la déformation totale et en compression les déformations positives sont obtenues en calculant les déformations transversales (effet de Poisson) à partir de la déformation longitudinale.

Les paramètres $\varepsilon_{0 \mathrm{t}}$ et $\varepsilon_{0 \mathrm{c}}$ sont des seuils en déformation d'apparition de l'endommagement, en traction et en compression. $A_{t}, B_{t}, A_{c}$ et $B_{c}$ sont quatre paramètres $\mathrm{du}$ modèle contrôlant la phase post-pic du comportement. L'identification de ces différents paramètres est établie à partir du calage des lois de comportement du matériau, lorsque celles-ci sont disponibles.

Dans le cadre du benchmark, ces paramètres ont été choisis ou déterminés comme suit :

- le seuil $\varepsilon_{0 \mathrm{t}}$ est égal au rapport de la résistance à la traction et du module élastique. La résistance à la traction est posée comme égale à $70 \%$ de la valeur obtenue par fendage,

- en l'absence de courbes de comportement en traction, nous avons choisi pour les paramètres $A_{t}$ et $B_{t}$ des valeurs classiques de la littérature,

- les paramètres $\varepsilon_{0 c}, A_{c}$ et $B_{c}$ ont été calés à partir des courbes issues des essais de compression. La figure 2 donne l'exemple du calage pour les courbes du béton de type 2.1, utilisé dans certaines poutres,

- la longueur caractéristique lc du matériau (paramètre permettant le calcul de la déformation équivalente) est prise de manière classique comme égale à trois fois le diamètre du plus gros granulats (25 mm) (Bazant et al., 1989).

Le tableau 1 récapitule pour chaque béton les données et paramètres utilisés dans le modèle. 


\begin{tabular}{|c|c|c|c|c|c|c|c|c|c|c|c|}
\hline \multirow{2}{*}{$\begin{array}{c}\text { Type } \\
\text { de } \\
\text { Béton }\end{array}$} & \multirow{2}{*}{$\begin{array}{c}\mathbf{R c} \\
(\mathbf{M P a})\end{array}$} & \multirow{2}{*}{$\begin{array}{c}\mathbf{R t} \\
(\mathbf{M P a})\end{array}$} & $\mathbf{E}(\mathbf{G P a})$ & $\mathbf{6}$ & $\begin{array}{c}\mathrm{lc} \\
(\mathrm{mm})\end{array}$ & $\begin{array}{c}\varepsilon_{0 \mathrm{t}} \\
\left(10^{-4}\right)\end{array}$ & $\mathrm{At}$ & $\mathrm{Bt}$ & $\begin{array}{c}\varepsilon_{0 \mathrm{c}} \\
\left(10^{-4}\right)\end{array}$ & $\mathrm{Ac}$ & $\mathrm{Bc}$ \\
\hline $\mathbf{1 . 1}$ & 49,5 & 3,5 & 34,7 & 0,2 & 75 & 1,01 & 1 & 30000 & 1,15 & 1 & 1025 \\
\hline $\mathbf{1 . 2}$ & 65 & 4,3 & 38,6 & 0,2 & 75 & 1,12 & 1 & 30000 & 1,12 & 1,3 & 1050 \\
\hline $\mathbf{2 . 1}$ & 44,5 & 4 & 30,5 & 0,2 & 75 & 1,31 & 1 & 30000 & 1,31 & 1,3 & 1250 \\
\hline $\mathbf{2 . 2}$ & 48,1 & 3,4 & 33,1 & 0,2 & 75 & 1,02 & 1 & 30000 & 1,31 & 1,3 & 1250 \\
\hline
\end{tabular}

Tableau 1. Données expérimentales et paramètres utilisés dans le modèle

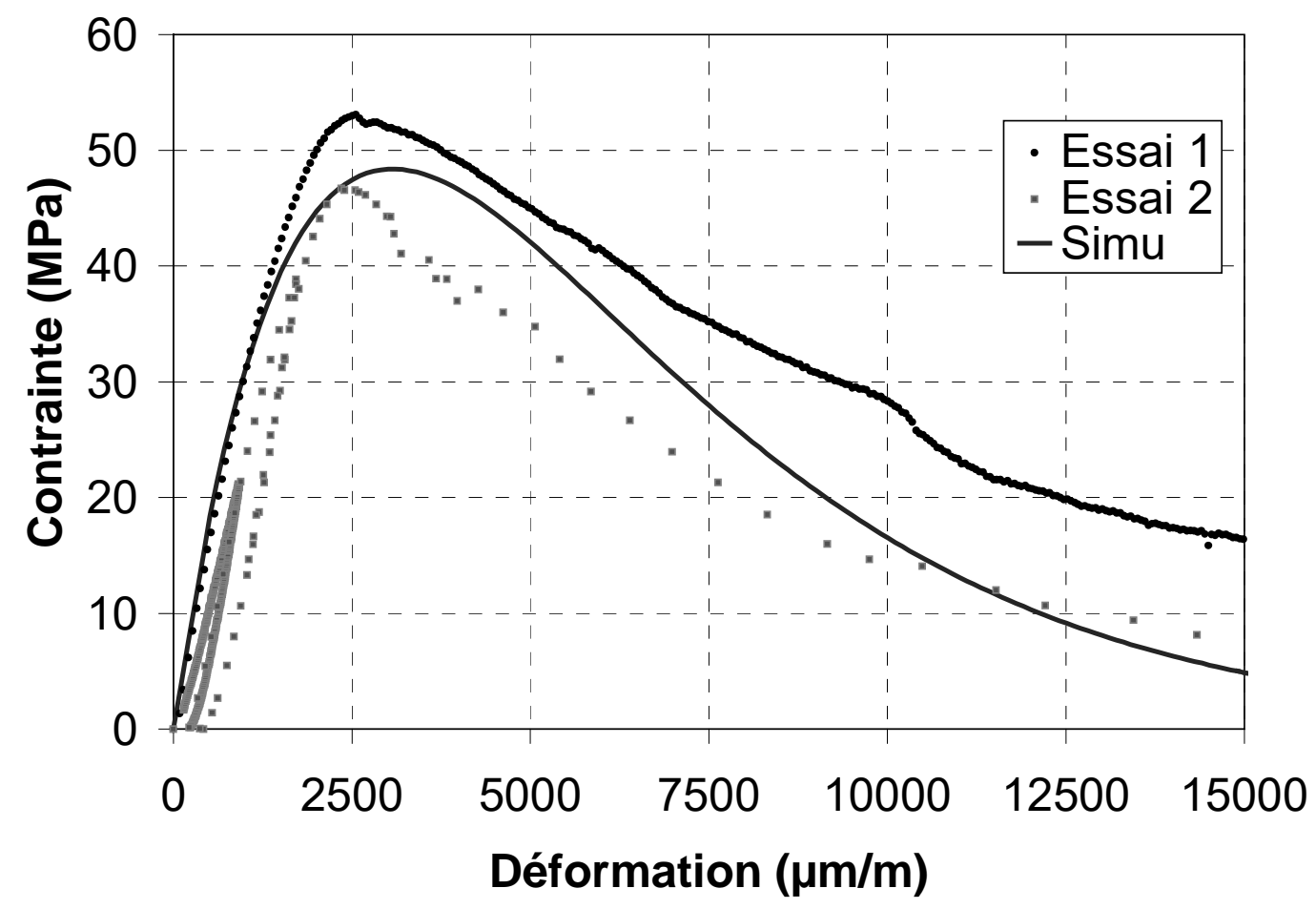

Figure 2. Calage des paramètres liés à la compression du béton de type 2.1

\subsection{Comportement de l'acier}

Le comportement des aciers passifs est un comportement élasto-plastique avec écrouissage positif. Les données ont été calées à partir des résultats expérimentaux obtenus par le CEBTP. Ces données sont répertoriées dans le tableau 2.

\begin{tabular}{|c|c|c|}
\hline Limite élastique (MPa) & Module d'élasticité (GPa) & Pente écrouissage (GPa) \\
\hline 309 & 195 & 0,7 \\
\hline
\end{tabular}

Tableau 2. Données relatives au comportement des aciers passifs 


\subsection{Modélisation de l'interaction acier/béton}

Les éléments de notre code de calcul sont des éléments en 1 dimension; la modélisation de l'interaction entre l'acier et le béton ne peut pas se faire, comme dans le cas des éléments en deux ou trois dimensions, en considérant le cisaillement au niveau de l'interface acier/béton. L'approche adoptée est donc phénoménologique. L'expérience montre que la rigidité d'une pièce en béton armé augmente avec l'adhérence entre le béton et les armatures. Dans Eficos, ce phénomène est modélisé par l'ajout d'une surface « interface » $S_{\text {ia }}$ dans la couche où se trouve l'acier (Feenstra, 1993). Le comportement de cette interface, donné sur la figure $3 b$, est constitué de trois phases : élastique, plastique et écrouissage négatif. Le module sécant de la couche est calculé par homogénéisation des trois constituants : béton, acier, interface (équation [12]).

$$
\mathrm{S}_{\mathrm{t}} * \mathrm{E}=\mathrm{S}_{\mathrm{a}} * \mathrm{E}_{\mathrm{a}}+\mathrm{S}_{\mathrm{b}} * \mathrm{E}_{\mathrm{b}}+\mathrm{S}_{\mathrm{ia}} * \mathrm{E}_{\mathrm{ia}}
$$

Cette modélisation a un fondement physique lorsque des aciers HA sont considérés. Il s'agit de représenter l'endommagement local du béton autour des nervures de l'armature. La largeur de l'interface est prise égale à la taille des nervures de l'acier (Lee et al., 2002). On considère que le comportement de l'interface acier béton $S_{\text {ia }}$ (figure 3a) est celui de l'acier non corrodé dans la phase élastique et au début de la phase plastique (figure 3b) (Feenstra, 1993). La limite élastique de cette interface est donc égale à celle de l'acier dans notre approche.

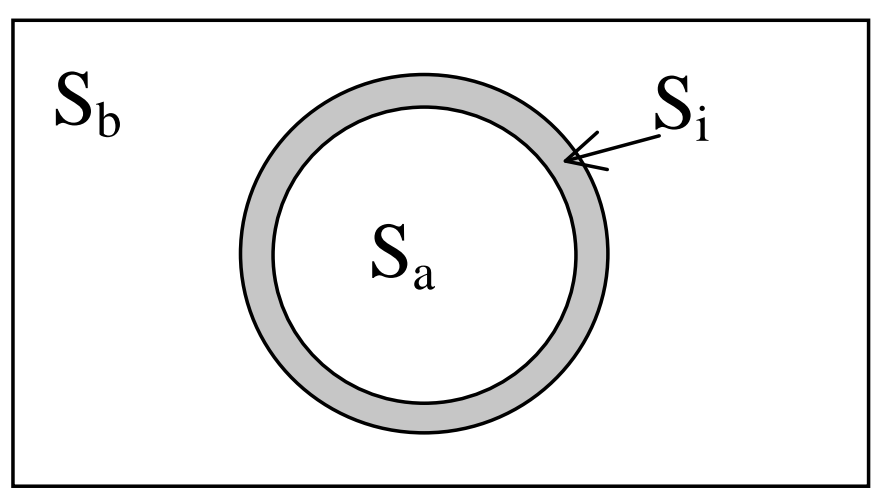

a)

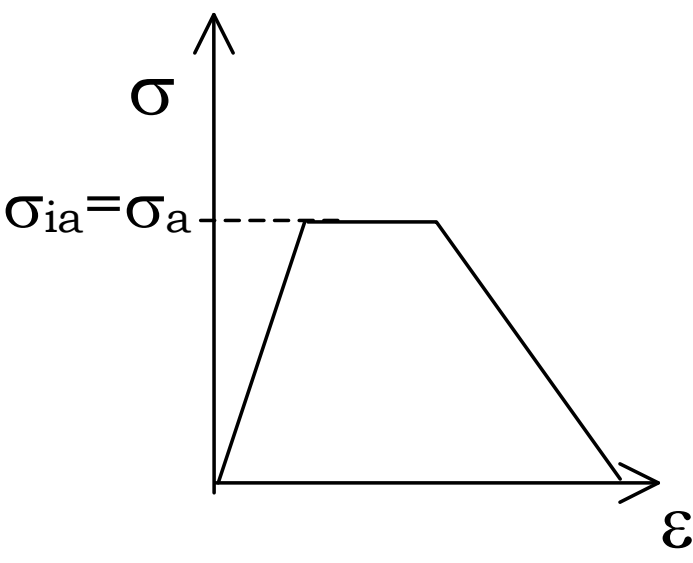

b) Comportement de l'interface Sia

Figure 3. Prise en compte de l'adhérence acier/béton

Cette modélisation de l'adhérence a été validée avec les résultats expérimentaux obtenus sur une poutre en béton armé B35 non corrodée et sollicitée en flexion (figure 4). Cette poutre de $35 \mathrm{~m}$ de long et de section $0,5^{*} 0,2 \mathrm{~m}^{2}$ est en béton B35 avec HA 500. Elle est similaire à celle testée dans le cadre du Benchmark MECA (Ghavamian et al., 2003). 


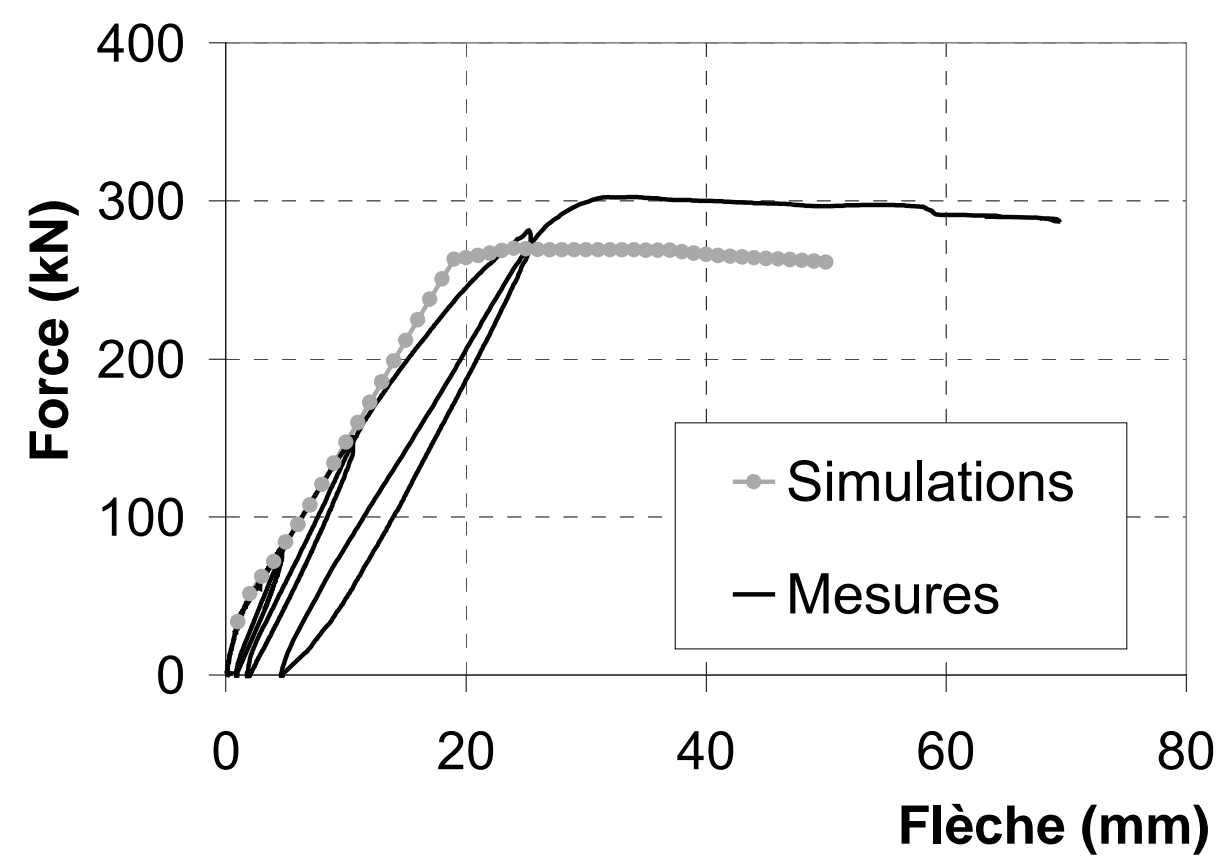

Figure 4. Simulation du comportement d'une poutre en béton armé avec prise en compte de l'adhérence

Dans le cas d'aciers lisses, le choix des paramètres de l'interface (Sia, limite élastique...) est plus délicat. Or les aciers passifs des poutres de la Rance sont des ronds lisses présentant de la corrosion. On peut supposer que l'adhérence acier/béton est faible dans nos poutres, d'autant plus que la corrosion a diminué les sections des armatures. C'est pourquoi nous avons choisi de ne pas prendre en compte l'augmentation de la rigidité due à l'adhérence : la surface interface $S_{\mathrm{ia}}$ est prise nulle dans nos calculs.

\subsection{Prise en compte de la corrosion}

Comme décrit précédemment, nous ne modélisons pas l'effet éventuel de l'interaction entre les armatures et le béton sur la rigidité de nos éléments finis. Nous négligeons de ce fait tout effet de la corrosion sur l'interaction acier/béton. Par conséquent, dans notre modèle, nous prenons en compte la corrosion en considérant uniquement les pertes de sections d'acier qu'elle a occasionnées.

La surface totale d'acier, à une hauteur donnée de la section de la poutre (figure 5), est calculée comme la somme des surfaces des aciers situés à cette hauteur. L'évolution de la surface totale d'acier ainsi obtenue suivant la longueur de la poutre est donnée à titre d'exemple sur la figure 6 (cas de la poutre P621). Ce profil est ensuite discrétisé suivant le nombre d'éléments choisis pour la simulation : la section d'acier dans chaque élément est la moyenne de la section sur la longueur de l'élément. Suite à cette discrétisation longitudinale, les sections d'acier peuvent être scindées en deux par la discrétisation en couches de l'élément. 
La qualité des simulations doit dépendre a priori du nombre d'éléments retenus ; Plus le nombre d'éléments est faible, par exemple, plus l'évolution longitudinale de la section d'acier est lissée. En réalité, il s'avère que dans le cas de nos poutres, le nombre d'éléments a peu d'importance, comme nous le montrons plus loin. La répartition de la corrosion est en fait suffisamment homogène pour que le comportement de la poutre ne soit pas affecté par la discrétisation.

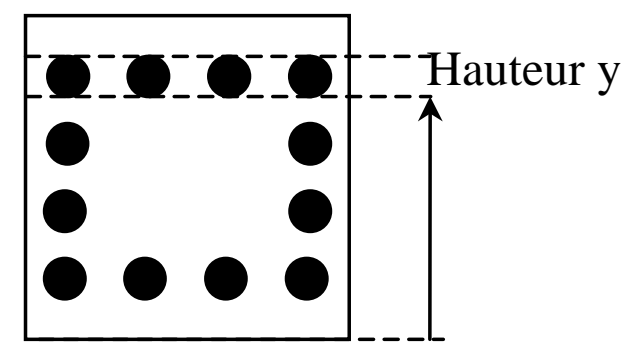

Figure 5. Section de la poutre

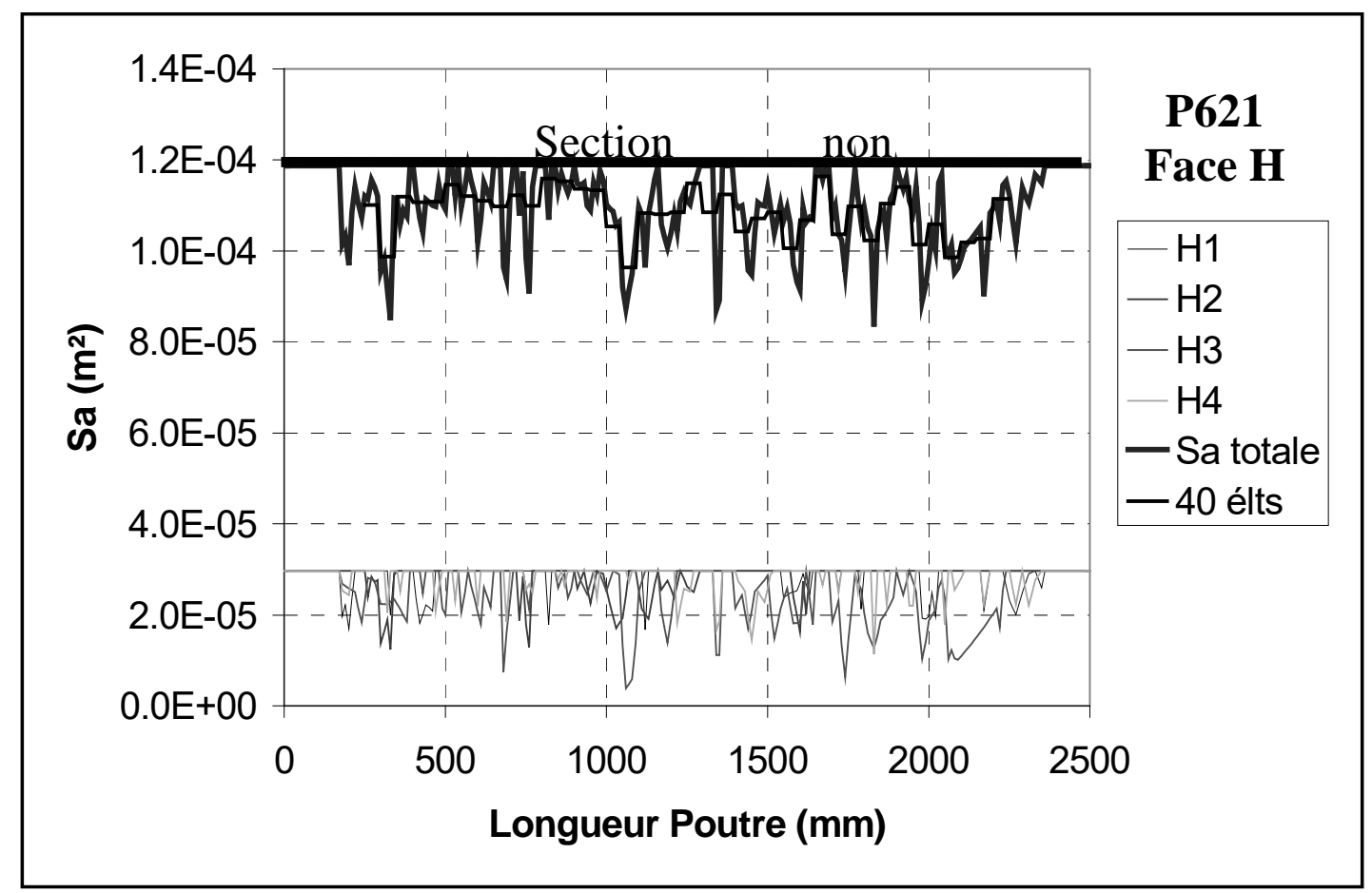

Figure 6. Prise en compte de la diminution de section d'acier pour la poutre 621

\section{Géométrie et conditions aux limites}

La poutre étudiée présente les dimensions suivantes : la longueur vaut $2 \mathrm{~m}$ avec

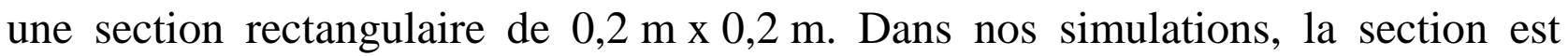
discrétisée en 18 couches horizontales. Le nombre d'éléments retenu est de 50 pour chaque poutre (éléments de taille constante). 
Les conditions aux limites en flexion sont les suivantes: pour le nœud 1, blocage des déplacements en $\mathrm{x}$ et $\mathrm{y}$ (rotule) et pour le nœud 51, blocage du déplacement en y (appui simple). Dans le cas de la traction, aucun déplacement n'est autorisé pour le nœud 1 alors que pour le dernier nœud le déplacement n'est autorisé que suivant $\mathrm{x}$ (figure 1). En traction, le chargement est imposé suivant $\mathrm{x}$ au dernier nœud. En flexion, le chargement est imposé suivant y aux nœuds 16 et 36, correspondant aux abscisses $\mathrm{x}=0,6 \mathrm{~m}$ et $\mathrm{x}=1,4 \mathrm{~m}$ (flexion 4-points).

La figure 7 présente différentes simulations du comportement d'une poutre du benchmark en fonction du nombre d'éléments choisis. Des instabilités apparaissent en dessous d'un certain nombre d'éléments. En fait, le nombre d'éléments est conditionné par le choix de la longueur $l_{c}$ (voir section 2.2) : la taille d'un élément doit être inférieure à $1_{c}$. Dans le cas contraire, l'endommagement n'est pas moyenné sur différents éléments (à l'instar de la déformation équivalente) et se localise successivement dans les éléments les plus sollicités. La longueur caractéristique de nos bétons étant de $75 \mathrm{~mm}$, le nombre d'éléments critique est de 27 . Au-delà de cette valeur, le comportement de la poutre n'est pas affecté par la discrétisation.

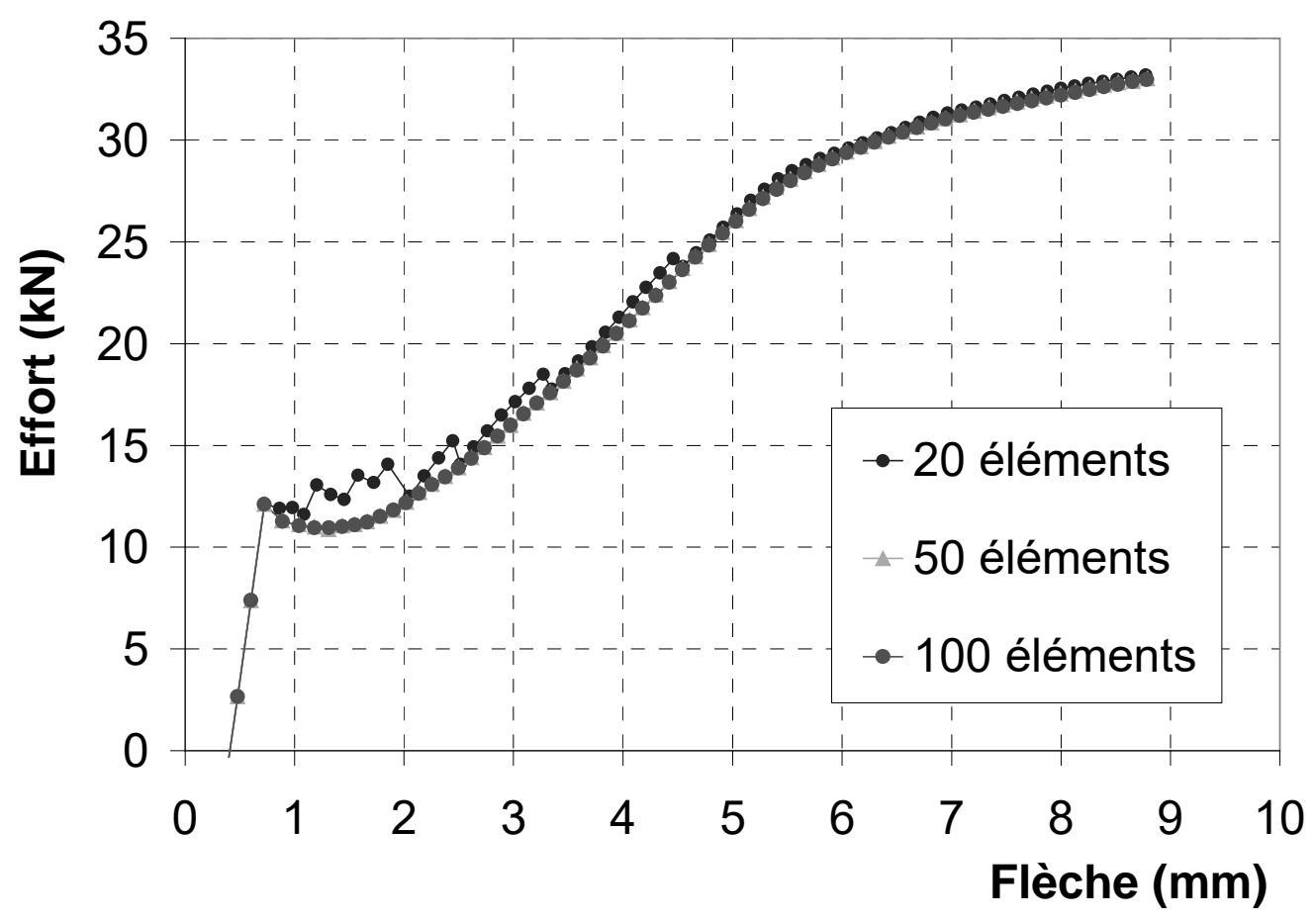

Figure 7. Influence du nombre d'éléments sur les simulations de la poutre 621 avec prise en compte de la corrosion

\section{Réalisation des calculs}

Le calcul est piloté en déformations et est stoppé lorsqu'il y a non-convergence pour des raisons numériques. En supposant que cette absence de convergence traduit une perte soudaine de portance par instabilité (de type snap-back), nous considérons dans la suite que la rupture est atteinte à la fin du calcul. 


\section{Résultats des simulations et comparaison avec les essais mécaniques}

Le récapitulatif des essais mécaniques effectués au CEBTP est donné tableau 3. Les simulations ont été effectuées pour chaque poutre et chaque type de chargement. Le comportement global force/déplacement est donné en considérant la poutre avec (poutre corrodée) et sans corrosion (poutre saine). Ces simulations sont comparées avec résultats expérimentaux obtenus au CEBTP (expérience) sur les poutres corrodées. Les essais du CEBTP sont effectués à déplacement imposé.

\begin{tabular}{|l|c|c|c|c|}
\hline Traction & 121 & 122 (cyclique) & $\begin{array}{c}211 \\
\text { (cyclique) }\end{array}$ & 212 \\
\hline $\begin{array}{l}\text { Flexion } \\
\text { Configuration BA }\end{array}$ & $\begin{array}{c}412 \\
\text { (cyclique) }\end{array}$ & $\begin{array}{c}611 \\
\text { (cyclique) }\end{array}$ & 621 \\
\hline $\begin{array}{l}\text { Flexion } \\
\text { Configuration BP }\end{array}$ & $\begin{array}{c}421 \\
\text { (cyclique) }\end{array}$ & $\begin{array}{c}622 \\
\text { (cyclique) }\end{array}$ & \multicolumn{2}{|c|}{911} \\
\hline
\end{tabular}

Tableau 3. Récapitulatif des essais mécaniques réalisés

Certaines poutres ont été testées avec une sollicitation cyclique. Les cycles n'ont pas été simulés, car le modèle de comportement retenu pour le béton, élastique endommageable, ne permet pas de rendre compte des déformations irréversibles ; un modèle élastique plastique endommageable serait plus approprié (Jason et al., 2006). Il faudrait aussi modéliser la refermeture de fissures (Fichant et al., 1999).

Rappelons que notre modèle ne permet pas de renseigner sur la position et l'ouverture des fissures. Les comparaisons entre simulations et essais mécaniques seront données à la section 6 .

\subsection{Essai de traction directe}

La figure 8 présente les résultats des simulations et la comparaison avec les essais mécaniques pour les poutres 121, 122, 211 et 212 sollicitées en traction directe.

\subsection{Essai de flexion 4-points (configuration béton armé)}

La figure 9 présente les résultats des simulations et la comparaison avec les essais mécaniques des poutres 412, 611 et 621 sollicitées en flexion 4-points. Ces poutres sont précontraintes; elles sont positionnées avec les câbles de précontrainte en zone comprimée, de manière à les solliciter dans une configuration «béton armé » (BA). 
P121

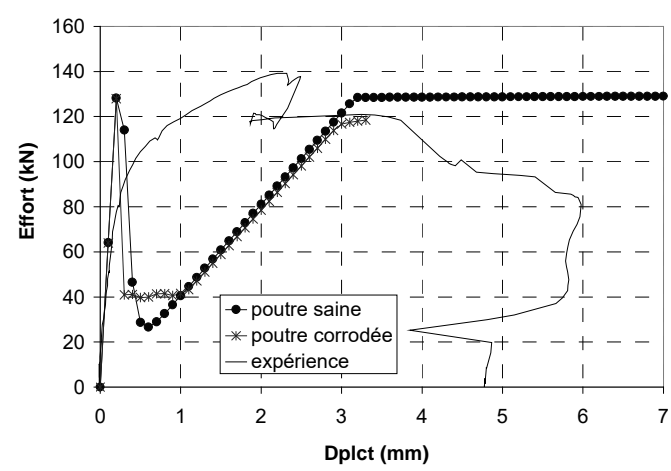

P211

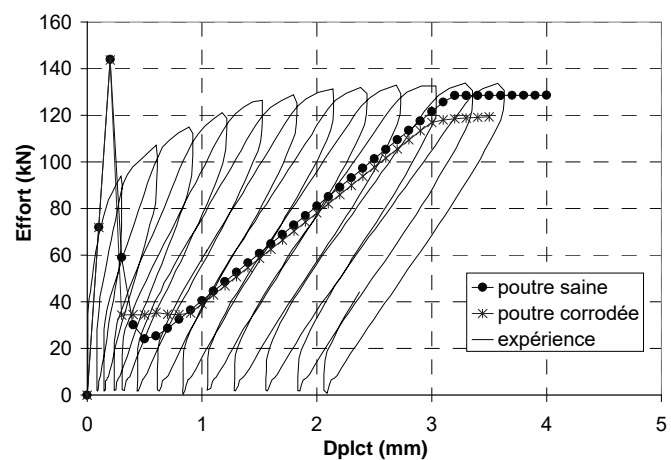

P122

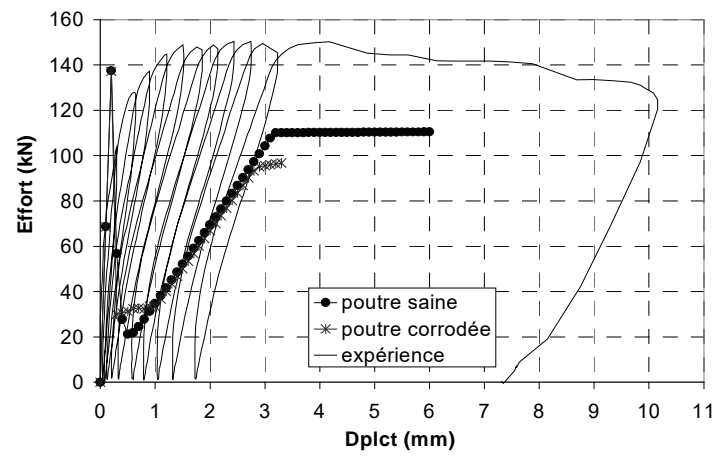

P212

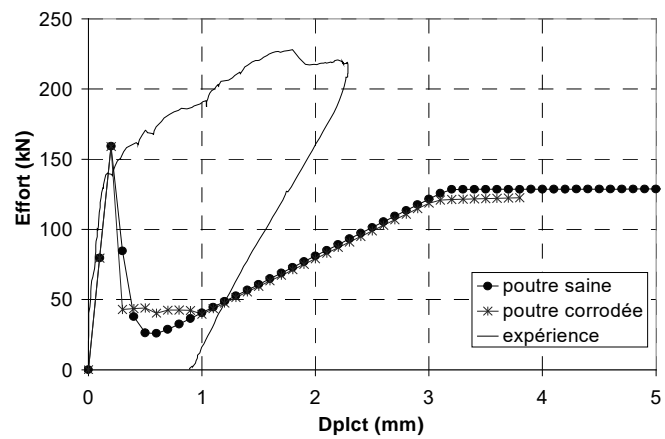

Figure 8. Comparaison simulations et expérimentations pour les poutres sollicitées en traction

P412

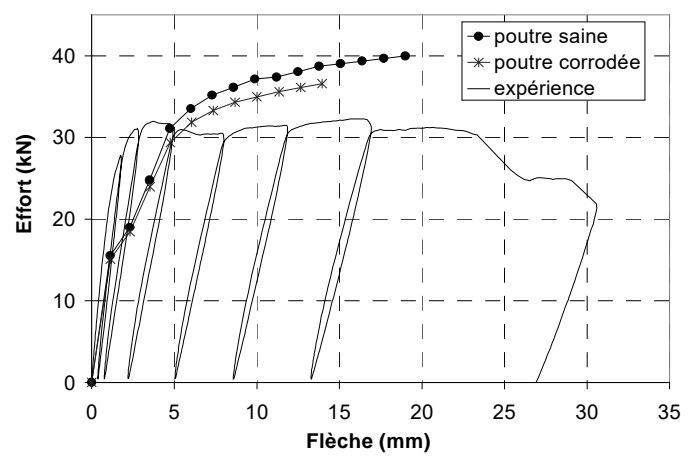

P611

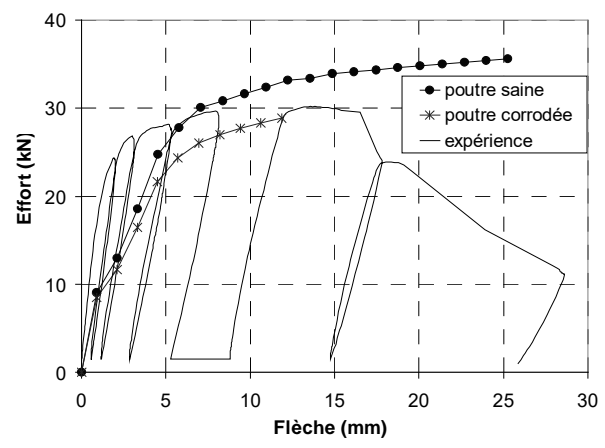

P621

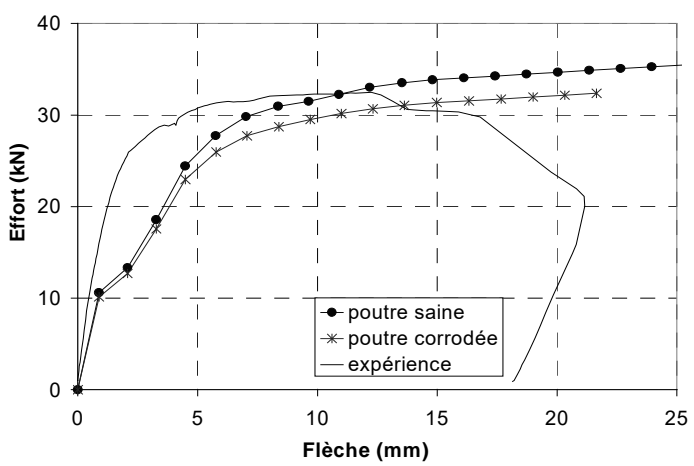

Figure 9. Comparaison simulations et expérimentations pour les poutres sollicitées en flexion (configuration $B A$ ) 


\subsection{Essai de flexion 4-points (configuration béton précontraint)}

La figure 10 présente les résultats des simulations et la comparaison avec les essais mécaniques des poutres 421622 et 911 sollicitées en flexion 4-points. Dans ce cas, les câbles de précontrainte sont placés en zone tendue afin de solliciter les poutres dans une configuration «béton précontraint » $(\mathrm{BP})$.

P421

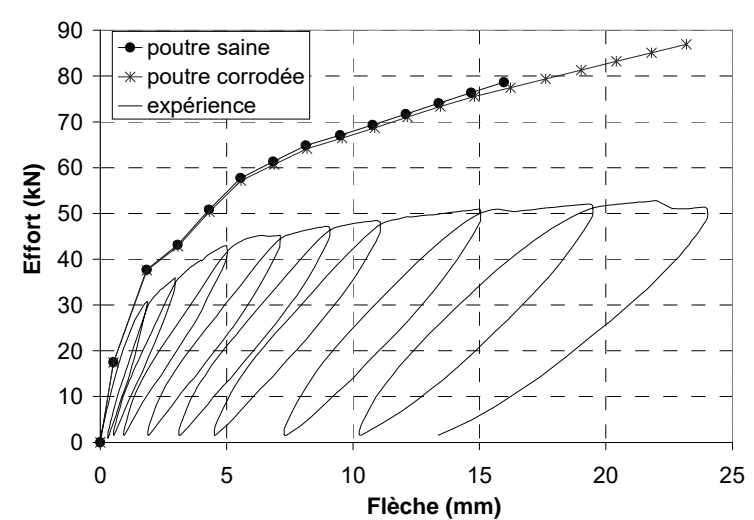

P622

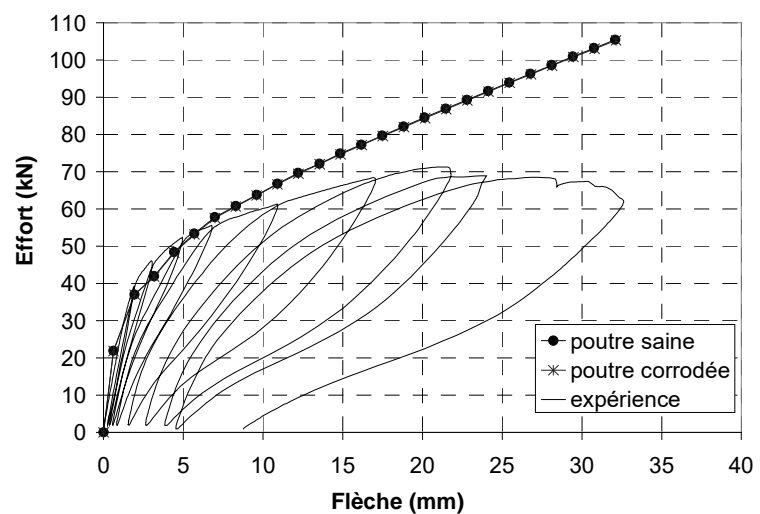

P911

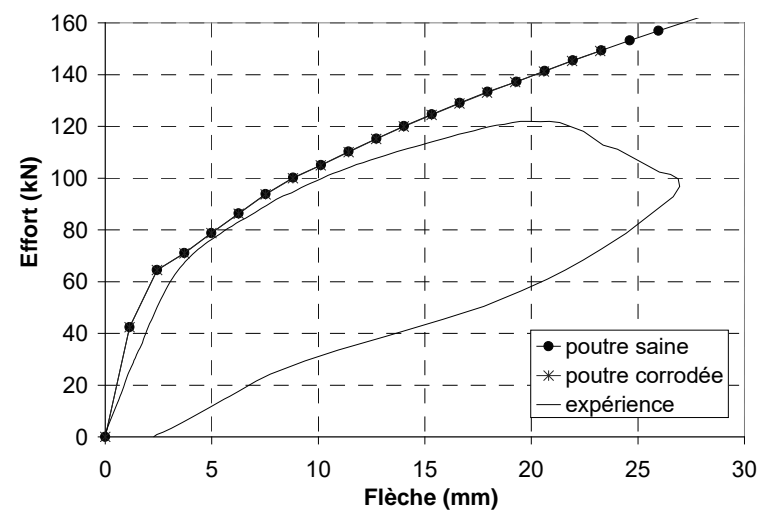

Figure 10. Comparaison simulations et expérimentations pour les poutres sollicitées en flexion (configuration béton précontraint)

\section{Discussion}

\subsection{Comparaison des simulations poutre saine et corrodée}

Les tableaux 4 et 5 présentent un récapitulatif des résultats obtenus en comparant le comportement des poutres corrodées et non corrodées. Les sections $S_{\mathrm{ac}}$ et $S_{\mathrm{anc}}$ représentent respectivement les sections d'acier corrodés et non corrodés de la couche la plus sollicitée (zone tendue). Les forces $F_{c}$ et $F_{n c}$ représentent respectivement les efforts atteints par les poutres dans le cas corrodé et non corrodé pour un déplacement de $4 \mathrm{~mm}$ pour les essais de traction et une flèche de $10 \mathrm{~mm}$ pour les essais de flexion. 


\begin{tabular}{|c|c|c|c|c|}
\hline & 121 & 122 & 211 & 212 \\
\hline $\mathrm{S}_{\mathrm{ac}} / \mathrm{S}_{\mathrm{anc}}(\%)$ & 97 & 93,4 & 95,8 & 96,9 \\
\hline $\mathrm{F}_{\mathrm{c}} / \mathrm{F}_{\mathrm{nc}}(\%)$ & 97 & 88 & 93 & 95,3 \\
\hline
\end{tabular}

Tableau 4. Récapitulatif quantitatif de l'influence de la corrosion (traction)

\begin{tabular}{|c|c|c|c|c|c|c|}
\hline & $\begin{array}{c}412 \\
(\mathrm{BA})\end{array}$ & $\begin{array}{c}611 \\
(\mathrm{BA})\end{array}$ & $\begin{array}{c}621 \\
(\mathrm{BA})\end{array}$ & $\begin{array}{c}421 \\
(\mathrm{BP})\end{array}$ & $\begin{array}{c}622 \\
(\mathrm{BP})\end{array}$ & $\begin{array}{c}911 \\
(\mathrm{BP})\end{array}$ \\
\hline $\mathrm{S}_{\mathrm{ac}} / \mathrm{S}_{\text {anc }}(\%)$ & 94 & 84 & 91 & 87 & 94,6 & 94,9 \\
\hline $\mathrm{F}_{\mathrm{c}} / \mathrm{F}_{\mathrm{nc}}(\%)$ & 95 & 89 & 93 & 99,2 & 99,7 & 99,8 \\
\hline
\end{tabular}

Tableau 5. Récapitulatif quantitatif de l'influence de la corrosion (flexion) l'adhérence

Dans le cas de la traction et de la flexion en configuration BA, le comportement mécanique des poutres est directement lié à la perte de section puisque les rapports $\mathrm{S}_{\mathrm{ac}} / \mathrm{S}_{\mathrm{anc}}$ et $\mathrm{F}_{\mathrm{c}} / \mathrm{F}_{\mathrm{nc}}$ indiqués dans les tableaux 4 et 5 sont proches. Ceci traduit l'hypothèse que seule la perte de section due à la corrosion influe sur le comportement mécanique des poutres corrodées (section 2.5). Cette correspondance pourrait être bien utile pour évaluer quantitativement le comportement mécanique d'une poutre corrodée.

La corrosion étant assez faible, elle n'affecte pas de façon significative le comportement des poutres. Notons également que la discrétisation choisie pour la prise en compte de la corrosion a tendance à lisser la corrosion le long de l'armature.

Dans le cas de la flexion en configuration BP (tableau 5 et figure 10), les câbles de précontrainte reprennent les efforts et la corrosion n'a quasiment pas d'influence sur le comportement mécanique des poutres.

\subsection{Comparaison simulations/essais mécaniques pour la poutre corrodée}

Rappelons que les essais réalisés au CEBTP sont effectués à déplacement imposé et que le calcul numérique est piloté en déformation.

\subsubsection{Cas de la traction}

Le premier pic de la courbe correspond au comportement du béton. On vérifie facilement que la pente initiale est proportionnelle au module élastique du béton et à 
sa section. De même, la charge au pic correspond approximativement à la résistance à la traction d'un béton (les paramètres du modèle d'endommagement en traction ont été choisis forfaitairement, comme indiqué section 2.2.)

Après le pic, la perte de portance assez brutale s'explique par l'endommagement complet du béton. La seconde phase de la courbe correspond au comportement de l'acier. On retrouve par exemple le module élastique et la section de l'acier dans la partie linéaire de cette phase.

Les courbes issues des simulations sont assez éloignées des résultats expérimentaux, notamment vis-à-vis de leur cinétique. Seules les pentes initiales des courbes (partie élastique) se superposent assez bien. Deux explications peuvent être fournies pour justifier de l'absence de concordance entre essais et simulations. D'abord, il semble que le comportement du béton simulé soit trop fragile. Les paramètres $\mathrm{At}$ et $\mathrm{Bt}$, qui gèrent le comportement en traction dans le modèle d'endommagement, n'ont pas été calés sur des essais en traction sur le béton testé. D'autres valeurs pour ces paramètres auraient permis de diminuer la fragilité du béton, et par conséquent de diminuer la perte de portance brusque en post-pic. Ensuite, les essais de traction réalisés au CEBTP ne sont pas des essais de traction pure, puisqu'une excentricité des efforts a été mise en évidence lors des expérimentations. Cette excentricité n'a pas été prise en compte dans nos simulations.

\subsubsection{Cas de la flexion (configuration $B A$ )}

Les simulations sont dans ce cas beaucoup plus proches des courbes expérimentales. La cinétique des courbes est globalement bien respectée, même si, comme évoqué précédemment, le choix du béton moins fragile aurait permis de rendre le pic initial (lié à la rupture en traction du béton) moins prononcé, et finalement de rapprocher les courbes théoriques des courbes expérimentales dans la phase post-pic. En outre, la pente finale des simulations est plus élevée que celle obtenue expérimentalement. Le plateau des courbes expérimentales avant la rupture correspond sans doute aux glissements à l'interface armatures/ béton, phénomène qui n'a pas été modélisé. Au final, la capacité portante des poutres théoriques est néanmoins du même ordre de grandeur que la capacité des poutres réelles.

\subsubsection{Cas de la flexion (configuration $B P$ )}

Mise à part la poutre 421, les simulations sont relativement proches des essais. Cependant, il semble que la poutre théorique soit là encore trop rigide lorsque la flèche devient élevée (après $10 \mathrm{~mm}$ ). Le comportement des poutres BP est surtout influencé par la précontrainte, les aciers jouant un rôle minime (on le constate avec l'absence d'effet de la perte de section due à la corrosion, section 6.1). On peut donc penser que la grande rigidité de la poutre théorique résulte d'un endommagement pas assez accentué, en particulier dans la zone comprimée du béton. 


\section{Conclusion}

Le code Eficos a été validé dans le cas d'une poutre en béton armé avec aciers HA non corrodés, sollicitée en flexion, avant d'être utilisé pour la simulation des poutres corrodées du benchmark. Rappelons que notre code n'a pas été utilisé pour simuler les cycles et qu'il ne peut donner aucune information sur la position et l'ouverture des fissures.

Seules les comparaisons simulations/expérience dans le cas de la flexion configuration BA donnent de bons résultats : on retrouve la pente initiale et la capacité portante. Dans le cas de la flexion configuration BP, le module initial est retrouvé mais la capacité portante est surestimée. Dans le cas de la traction, les modules élastiques sont retrouvés alors qu'on note une absence de concordance entre les cinétiques dues à la fragilité du béton.

Le modèle d'endommagement utilisé est un modèle qui emploie des paramètres qui doivent être calés à partir des courbes contraintes déformations obtenus à partir des essais de compression et traction sur les bétons. Ces essais ont été réalisés pour la compression, d'où la détermination précise des paramètres $\mathrm{Ac}$ et $\mathrm{Bc}$ mais non pour la traction où seule la contrainte à la rupture a été déterminée. Les résultats de simulations pourraient donc être améliorés si les paramètres de traction étaient calés précisément. Dans le cas de la traction et de la flexion configuration BA, l'obtention d'un béton moins fragile aurait permis une meilleure concordance en phase post-pic.

Un autre facteur peut expliquer les valeurs élevées de capacité portante dans le cas des simulations. En effet seule la perte de section a été introduite dans notre modèle pour prendre en compte la corrosion, or le glissement acier/béton doit également être pris en compte même avec des ronds lisses.

Dans le cas de la traction, le modèle peut être amélioré en prenant en compte l'excentricité de la précontrainte qui a été évaluée expérimentalement.

\section{Remerciements}

L'équipe du GeM remercie vivement toute l'équipe du benchmark pour le travail effectué et les collaborations établies au cours de cette étude.

\section{Bibliographie}

Bazant Z.P., Pan J.Y., Pijaudier-Cabot G., "Strain-softening in reinforced concrete beams and frames", Journal of structural engineering, vol. 113, n 12, 1987, p. 2333-2347.

Bazant Z.P., Pijaudier-Cabot G., "Measurement of the characteristic length of nonlocal continuum", Journal of engineering mechanics, vol. 115, n 4, 1989, p. 755-767. 
Feenstra P.H., Computational aspects of biaxial stress in plain and reinforced concrete, Thèse de doctorat, T.U. Delft, 1993.

Daniel L., Comportement de poutres en béton à hautes performances fibré sous sollicitations cycliques, Thèse de doctorat de l'Ecole Centrale de Nantes, 2001.

Fichant S., La Borderie C., Pijaudier-Cabot G., "Isotropic and anisotropic descriptions of damage in concrete structures", Int. J. mechanics of cohesive frictional materials, vol. 4, 1999, p. 339-359.

Ghavamian S., Delaplace A. (Eds.), « Modèles de fissuration de béton », Revue Française de Génie Civil, vol. 7, n 5, 2003.

Jason L., Huerta A., Pijaudier-Cabot G., Ghavamian S., "An elastic plastic damage formulation for concrete: application to elementary and comparison with an isotropic damage model", Computer methods in applied mechanics and engineering, vol. 195, $\mathrm{n}^{\circ} 52,2006$, p. 7077-7092.

La Borderie C., Phénomènes unilatéraux dans un matériau endommageable : modélisation et application à l'analyse des structures en béton, Thèse de doctorat, Ecole Normale Supérieure de Cachan, 1991.

Pijaudier-Cabot G., Mazars J., "Damage models for concrete", Handbook of materials behavior models, 2001.

Lee H.S., Noguchi T., Tomosawa F., "Evaluation of the bond properties between concrete and reinforcement as a function of the degree of reinforcement corrosion", Cement and Concrete Research, vol. 32, 2002, p 1313-1318. 